\title{
THE AUTOMATA THEORY OF SEMIGROUP EMBEDDINGS
}

\author{
MICHAEL ARBIB \\ (Received 5 September 1967)
}

\section{Abstract}

The theorem of Trevor Evans [1] that every countable semigroup can be embedded in a two-generator semigroup becomes obvious in automata theory as the statement that every countable automaton can be embedded in one with binary inputs. Standard techniques of automata theory [1], [3] yield a proof of the Evans Theorem using wreath products, as in Neumann [4].

\section{2.}

An automaton $M$ (without output) is a triple $(Q, X, \delta)$ where $Q$ is the set of states, $X$ is the input set, and $\delta: Q \times X \rightarrow Q$. The semigroup $S(M)$ is the subsemigroup of $Q^{Q}$ generated by the maps $\delta(\cdot, x): Q \rightarrow Q . M$ is called countable [finite] if $Q$ is countable [finite].

Given a countable semigroup $S$ with generators $G(S)$, we may represent it as the semigroup of the machine $M_{S}=\left(S^{1}, G(S), \delta_{S}\right)$ where $\delta_{S}$ is multiplication in the semigroup $S$, and $S^{1}$ is $S$ with a unit adjoined only if $S$ is not a monoid.

We then replace $M_{S}$ by a machine which has input set $\{0,1\}$ and which reads in strings until a code (i.e., a monomorphism of $\mathscr{F}_{G(S)}$, the free semigroup generated by $G(S)$, into $\left.\mathscr{F}_{\{0,1\}}\right)$ for an element of $G(S)$ has been read, and then acts accordingly.

We now give two examples of codes and the corresponding constructions.

One which works whether or not $G(S)=\left\{s_{1}, s_{2}, \cdots\right\}$ is finite is to code $s_{j}$ as $1^{j} 0$, i.e. a string of $j$ ones followed by a zero. Then

with

$$
M_{1}=\left(N \times S^{1},\{0,1\}, \delta_{2}\right) \quad(\text { taking } N=\{1,2,3, \cdots\})
$$

$$
\begin{aligned}
& \delta_{1}((n, s), 0)=\left(0, s \cdot s_{n}\right) \\
& \delta_{1}((n, s), 1)=(n+1, s)
\end{aligned}
$$

and the map $s_{j} \rightarrow 1^{j} 0$ yields an embedding of $S$ in the twc-generator 
semigroup $S\left(M_{1}\right)$. If $G(S)=\left\{s_{1}, \cdots, s_{d}\right\}$ is finite, we can replace $N$ by $\{1, \cdots, d\}$, addition then being modulo $d$ to handle sequences not coding words of $S$, so that $S\left(M_{1}\right)$ is finite if $S$ is finite.

If $G(S)=\left\{s_{1}, \cdots, s_{d}\right\}$ is finite and $2^{m-1}<d \leqq 2^{m}$ we may give a 'faster' construction, encoding each $s_{j}$ as a distinct string $t_{j}$ of $m 0$ 's and l's. Then if $A$ is the set of strings of at most $m-10$ 's and l's (including the empty string $A$ ) we may set

with

$$
M_{2}=\left(A \times S^{1},\{0,1\}, \delta_{2}\right)
$$

$\delta_{2}\left(\left[u_{1} \cdots u_{k}, s\right], u\right)= \begin{cases}{\left[u_{1} \cdots u_{k} u, s\right]} & \text { if } 0 \leqq k<n-1 \\ {\left[\Lambda, s \cdot s_{j}\right]} & \text { if } k=n-1 \text { and } t_{j}=u_{1} \cdots u_{k} u \\ {[\Lambda, s]} & \text { else }\end{cases}$

and the map $s_{j} \rightarrow t_{j}$ yields an embedding of $S$ in the two-generator semigroup $S\left(M_{2}\right)$.

It should be clear how this construction can be extended to arbitrary codes.

We now show how our result yields Neumann's wreath-product approach. The cascade $A_{2} \times Z_{Z}^{\eta} A_{1}$ of any two automata $A_{j}=\left(Q_{j}, X_{j}, \lambda_{j}\right)$ with connecting map $Z: Q_{1} \times X \rightarrow X_{2}$ and encoder $\eta: X \rightarrow X_{1}$ is the automaton with $\lambda_{Z}^{\eta}\left(\left[q_{2}, q_{1}\right], x\right)=\left[\lambda_{2}\left(q_{2}, Z\left(q_{1}, x\right)\right), \lambda_{1}\left(q_{1}, \eta(x)\right)\right]$ and (as was first pointed out by Krohn and Rhodes [3], see also [1]) the semigroup of $A_{2} \times{ }_{Z}^{\eta} A_{1}$ can be embedded in the wreath product of $S\left(A_{2}\right)$ and $S\left(A_{1}\right)$.

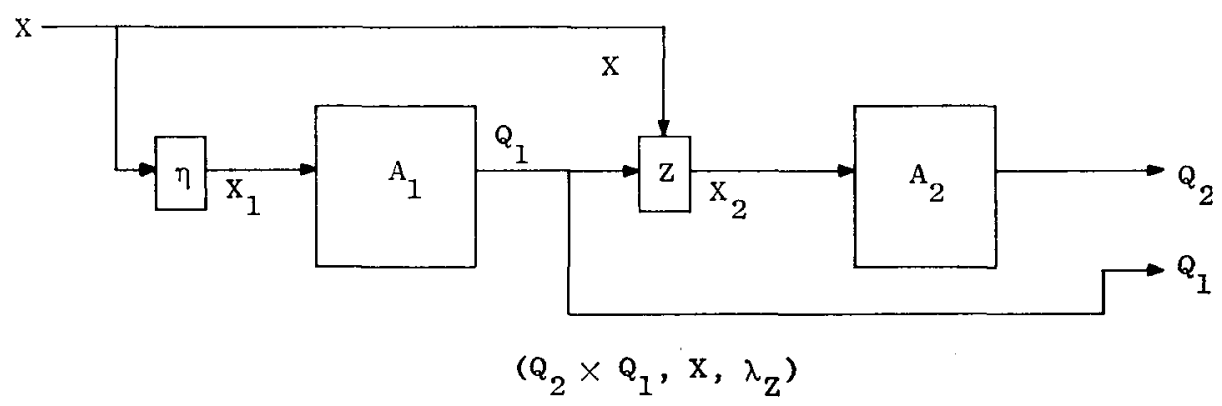

Now it is clear from the definitions of $\delta_{1}$ and $\delta_{2}$ that both $M_{1}$ and $M_{2}$ are cascades of some machine $A_{1}$ with $M_{S^{1}}$. In the case of the first construction, the semigroup $T_{0}$ of $A_{1}$ is $N^{0}\left[\{1, \cdots, d\}^{0}\right.$ if $G(S)$ is finite $]$ - that is, the integers under addition [modulo $d$ ] with a multiplicative zero adjoined. In either case, we see that $S$ can be embedded in a two-generator semigroup which can in turn be embedded in the wreath product of $S\left(A_{1}\right)$ and $S^{1}$. The first construction yields what is essentially Neumann's proof. However, where Neumann [4] took the order $m$ of his cyclic group $T$ to be at least $3 d$ 
in the finite case, we only need take $m=d$, though we must adjoin our 0 to $T$ whereas he adjoins his zero to $S$. It then becomes a simple exercise to obtain the results of Neumann's Section 5 with improved bounds. We hope this note will encourage further applications of automata theory to the algebraic theory of semigroups.

This research was supported in part by the U. S. Air Force Office of Scientific Research (Information Sciences Directorate) under Contract No. AF-AFOSR-1198-67.

My thanks to Shafee Give'on for a helpful reading of the manuscript.

\section{References}

[1] M. A. Arbib, 'Automaton Decompositions and Semigroup Extensions', in: The Algebraic Theory of Machines, Languages and Semigroups, M. A. Arbib (Editor), Academic Press, (1968), 37-54.

[2] T. Evans, 'Embedding Theorems for Multiplicative Systems and Projective Geometries', Proc. Amer. Math. Soc. 3 (1952), 614-620.

[3] K. Krohn and J. L. Rhodes, 'Algebraic Theory of Machines, I: The Decomposition Results', Trans. Amer. Math. Soc. 116 (1965), 450-464.

[4] B. H. Neumann, 'Embedding Theorems for Semigroups', J. London Math. Soc. 35 (1960), $184-192$.

\section{Electrical Engineering Department}

Stanford University

Stanford, California 94305 U.S.A. 